\title{
Chiasma interference is blind to centromeres*
}

\author{
P. C. COLOMBO $\$$ \& G. H. JONES†§ \\ $\ddagger$ Depto. de Cs. Biológicas, Facultad de Ciencias Exactas y Naturales, Universidad de Buenos Aires, (1428) Buenos \\ Aires, Argentina and \$School of Biological Sciences, The University of Birmingham, Edgbaston, Birmingham B15 \\ $2 T T, U . K$.
}

\begin{abstract}
It has long been assumed, since the early works of Mather, that the centromere plays a central role in chiasma position determination, so much so that in all sequential models chiasma determination was supposed to start or finish at this point. More specifically, it has been asssumed that the centromere acts as a barrier to the transmission of interference, so that a chiasma in the vicinity of a centromere would not affect the probability of chiasma formation across this point. Some statistical analyses seemed to ratify this supposition. However, a reassessment of the literature led us to the conclusion that the statistical analyses that were not flawed were consistent in showing that interference may act across the centromere. Using large sets of chiasma data from the grasshoppers Leptysma argentina and Chorthippus brunneus and applying statistical approaches that involved either the calculation of coincidence or correlating the distances between the centromere and the nearest chiasma in either arm, it is concluded:

1 that interference acts across the centromere;

2 that the action of interference is not changed by the presence of an intervening centromere.
\end{abstract}

Keywords: centromere, chiasmata, interference, Orthoptera.

\section{Introduction}

Interference, as originally conceived by Sturtevant (1915) and Muller (1916), was defined in purely genetic terms as a departure from independence of recombination in two chromosome regions. It was traditionally quantified in terms of coincidence $(C)$, which measures the degree of independence of the two regions of the chromosome under consideration. $C$ is calculated as the actual number of double recombinants divided by the expected number on the assumption of independence of recombination in the two regions. Generally, $C$ tends to 1 as the distance separating the two regions considered grows longer. Interference, according to this approach, is defined as $1-C$. Morgan (1919; cited by Whitehouse, 1965) called interference 'the fifth law of heredity' and it has been found in most organisms ever since.

Interference emerged, then, as a genetic concept. However, the chromosome theory of heredity and the growing consensus around the chiasmatype

\footnotetext{
*This paper is dedicated to Professor Dr J. H. Hunziker on the occasion of his 70 th birthday.

†Correspondence. E-mail: g.h.jones@bham.ac.uk
}

theory, which equated chiasmata with crossovers, in the mid-1930s forced cytologists and geneticists to a synthesis (see review in Whitehouse, 1965). The convergence between Haldane (1931; using cytological data of Maeda, 1930, from Vicia faba) and Mather (1933; using his own genetic data from Drosophila), paved the way for the most authoritative link between the two fields so far, a model for the operation of interference (Mather, 1938) that has remained largely unchallenged up to the present time.

Recent years have seen a revival of interest in this question, with the emergence of several models for the operation of interference (King \& Mortimer, 1990; Foss et al., 1993; Lande \& Stahl, 1993), many of which took for granted Mather's model (1938) and the slight modifications of his followers (especially Henderson, 1963; Southern, 1967 and Fox, 1973). Given the renewed importance of this issue we considered it an opportune time to reassess critically the available evidence concerning interference. We have found that many of the recent models use as premises cytological conclusions which have not been examined critically for decades. The re-examination of a large set of data from Chor- 
thippus brunneus taken from Laurie (1980), together with data from Leptysma argentina (partially published in Colombo, 1993) has allowed us to challenge one of the principal premises, namely the absence of interference across the centromere.

\section{Interference across the centromere}

One may be forgiven for thinking that, when it comes to crossover position determination, the centromere has supernatural properties of some sort. In a series of papers between 1936 and 1940, Mather (1936a,b, 1937, 1938, 1940) proposed a model of chiasma formation in which chiasma determination proceeded in a linear sequential way, starting at the centromere. According to his model (reviewed in Mather, 1938), the distance between the centromere and the first chiasma, which he called the differential distance $(d)$, is characteristic of every chromosome. The distance between all subsequent chiasmata is determined by interference, and thus it was called the interference distance $(i)$. This model was put forward as a hypothesis and remained untested for almost three decades. The first test came from Henderson (1963), who conducted the first detailed quantitative study of chiasma distribution at diplotene in Schistocerca gregaria, the karyotype of which is telocentric (therefore no analysis of chiasma interference across the centromere was possible). Henderson concluded from chiasma distribution patterns and the chiasma frequency/chromosome length relationship that chiasma formation was indeed sequential, but, on the grounds of the high distal chiasma frequency, he proposed that it proceeded towards the centromere. The same conclusion was drawn by Southern (1967), from his study of four Truxaline grasshopper species (the karyotype of which comprises three meta/ submetacentric and five telocentric pairs), and by Fox (1973), again in S. gregaria. However, Fox recognized that 'interference distance' was a misnomer, because interchiasma distance $(i x)$ is determined not only by interference but by other factors as well. According to Fox, interference determines the minimum interchiasma distance $\left(i x_{\min }\right)$ only, although this is also an oversimplification (Jones, 1987). Fox (1973) assumed, on the grounds of chiasma distribution, that chiasma sequential determination travelled from the telomere towards the centromere, incarnated in a 'chiasma determination mechanism' (CDM), which moves along the bivalent and triggers chiasmata. After having triggered a chiasma the CDM needs some time to recharge, this resulting in interference.
Given that the centromere is a differentiated structure whose function is to ensure proper orientation and segregation of the bivalents in metaphase and anaphase, what could its role in chiasma position determination be? For a better understanding of the origin of the remarkably central role proposed for the centromere in chiasma position determination, it is helpful to follow precedent and divide, quite artificially perhaps, the different approaches to crossover/chiasma distribution into two: genetic and cytogenetic. At the time Mather's model was put forward, the chiasmatype theory, which equates crossovers with chiasmata, had already been widely accepted, and hence the terms 'crossover' and 'chiasma' will be used interchangeably in the discussion that follows.

\section{Genetic approach}

Following the first description of genetic interference by Muller (1916) in terms of coincidence, the subject of crossover distribution control was at first wholly conducted in Drosophila, with genetic methodology and from a genetic point of view (Weinstein, 1918; Stevens, 1936; Charles, 1938). Following Haldane's (1931) precedent, Mather took the first steps towards the establishment of a unified theory of crossing-over, which reconciled both the cytological and genetic data known at that time. Mather (1936a) compared the genetic and cytogenetic (mitotic and salivary gland) maps of Drosophila and he noticed two features:

1 there was a disproportionate clustering of markers around the centromere in the genetic map when compared to the cytological map;

2 this was not caused by an effect of the telomeres, because the $\mathrm{X}$ chromosome (an acrocentric) was comparable to an arm of the metacentrics 2 and 3, and not to the whole chromosome.

Hence Mather hypothesized that the centromere had a causal relationship in the determination of crossing-over. However, he realized that the clustering of markers was not enough to demonstrate it, and so performed several critical experiments. Working with different inversions of Drosophila melanogaster, which positioned the markers closer or farther from the centromere and/or the pericentric heterochromatin of the $\mathrm{X}$ chromosome, he was able to conclude that: (i) crossing-over in the euchromatin was dependent on the distance to the centromere; and (ii) this was independent of the position of the heterochromatin (Mather, 1939). He then concluded that the centromere had a central role in the determination of crossovers. 


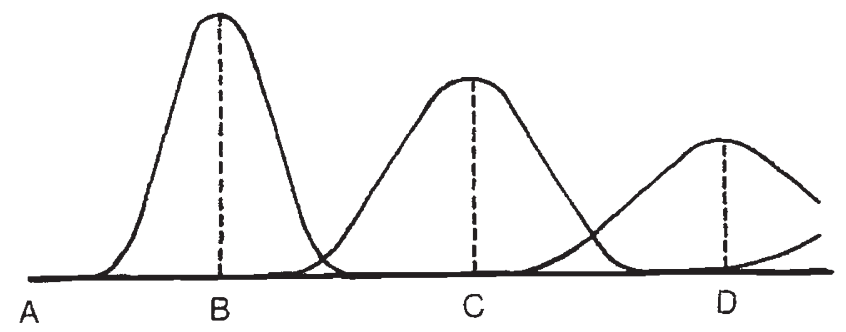

Fig. 1 Theoretical crossover distribution on the assumption of a linear sequential model (from Mather, 1938). A marks the position of the centromere and B, C and D the mean positions of the first, second and third formed chiasmata, assuming that chiasma formation proceeds sequentially from the centromere.

In order to test this hypothesis, he conducted a series of experiments in Drosophila. The purpose was to measure the variance of the 'first' chiasma with that of 'subsequent' chiasmata (despite the inverted commas we can see he assumed a sequence beforehand). The rationale behind this was that he deemed chiasma formation to be sequential, with the centromere as the starting point. If this were true, the predictable pattern of chiasma formation would be as shown in Fig. 1. The first chiasma would show a variance, inherent to the 'differential' distance $\left(\sigma_{d}\right)$; the second would show the inherent variance of the 'interference' distance plus the variance of the first chiasma $\left(\sigma_{d}^{2}+\sigma_{i 1}^{2}\right)$. The variance of the position of subsequent chiasmata would include their inherent variation and that attributable to the preceding ones $\left(\sigma_{d}^{2}+\sigma_{i 1}^{2}+\sigma_{i 2}^{2}+\ldots\right)$. This produces increasingly dispersed chiasma distributions (Fig. 1).

Mather could only test the 'first' and the 'second' chiasmata ('thirds' and 'fourths' were not frequent enough to perform statistical analyses), and this fitted his predictions for chromosomes X, II and III of Drosophila. Therefore, he put forward the linear sequential model of chiasma formation described above, with the centromere as the starting point (reviewed in Mather, 1938).

If chiasma formation was to be sequential and starting at the centromeres, then as a logical consequence the two arms of metacentric or submetacentric chromosomes should behave independently. At that time, Mather (1936a) could find plenty of evidence in favour of this hypothesis. In 1932 Beadle had performed a study on a III-IV translocation homozygote of $D$. melanogaster. In this translocation the bulk of the right arm of chromosome III had become attached to the short arm of chromosome IV. Beadle (1932) found that the regions of chromo- some III close to the translocation breakpoint showed a significant decrease of recombination when compared to the normal flies. He then concluded that the centromere had a significant role in the control of crossing-over. When he sought to explain this reduction he invoked Sax's (1930) model of recombination, which assumed independence of the arms. Beadle calculated coincidence between regions $1-6,2-6,1-7$ and $2-7$ and found levels of coincidence not significantly different from 1 (closer segments could not be compared as a result, precisely, of the low frequency of recombination around the centromere), which Beadle regarded as wholly expected according to the model of Sax (1930, 1932). To this very day Beadle (1932) is quoted as the first demonstration of lack of interference across the centromere (e.g. Foss et al., 1993).

The explanation for the disproportionate clustering of markers in Drosophila (the reduced amount of recombination, or of chiasma frequency) around the centromere, now, 60 years later, seems self-evident. It is the consequence of distal chiasma localization, one of the causes of departure from random chiasma distribution. In fact, chiasma localization, along with the obligate chiasma per bivalent and interference are the three main causes for the departure from Poisson expectations (see reviews in Jones, 1984, 1987). In fact, even in organisms where localization is not absolute, many species show a tendency towards reduced chiasma formation around the centromeres. This is far from contradictory to the title and the aim of this review. We do not mean to say that chiasma formation is blind to centromeres; on the contrary, there is profuse evidence that chiasma formation is very mindful of the position of the centromere (witness chiasma distribution in $C$. brunneus, below). What we do mean is that interference is blind to the centromere. We hope the difference will be clear throughout the paper. The first is a result of chiasma localization and can be evident even in bivalents with only one chiasma; the second is interference and needs at least two chiasmata: interference, by definition, cannot otherwise be detected.

To reiterate, an important reason for the proposed central role of the centromere in this context is that interference across the centromere was not detected in Beadle's (1932) work; the word detected is crucial. As a result of distal localization, many organisms, including Drosophila, show reduced frequency of crossovers around the centromere. Hence the regions close to the centromere (the only ones that could tell us something about this subject) 
do not have enough crossovers to perform meaningful statistical analyses. The regions compared by Beadle $(1-6,2-6,1-7,2-7)$ were simply too far apart to interact.

Stevens (1936) is often quoted in the literature as providing the definitive proof that interference does not operate across the centromere. He criticized earlier studies by Graubard (1932) and Schweitzer (1935) on the charges of inconsistency, and used yet another estimator of coincidence that failed to detect interference across the centromere in $D$. melanogaster. Mather (1936a) quoted Stevens (1936) as a watershed; Henderson (1963), Southern (1967) and Fox (1973) quoted Mather (1936a,b, 1938, 1940) and the whole subject was considered closed. Even quite recent discussions of interference unanimously take for granted that there is no interference across the centromere (King \& Mortimer, 1990; Foss et al., 1993; Lande \& Stahl, 1993; Munz, 1994). However, there were some reports, mainly coming from cytological studies, which succeeded in detecting interference across the centromere. We will deal with them in the next section, for enough has been said about genetic studies. Let us only mention that Pätau (1941) reassessed a very early recombination study of Gowen (1919) in D. melanogaster, by using the very consistent method of Stevens (1936), and succeeded in detecting interference across the centromere, but with a few notable exceptions (Callan \& Montalenti, 1947; Callan, 1949) this was not widely recognized.

\section{Cytological approach}

Much of the cytological evidence for the action of interference has been reviewed by Sybenga (1975). According to this author, there are three main approaches to this problem: (i) indirectly, by comparing chiasma frequencies per bivalent with random (in the restricted sense of Poissonian) expectations; (ii) by correlating numbers of chiasmata in relatively large chromosome segments such as whole chromosome arms (interference across the centromere) or segments of interchange heterozygote multiples; and (iii) by a more direct approach, which involves accurate measurements of chiasma distribution within bivalents in highly favourable material (this usually means grasshoppers and locusts). The first two approaches will be briefly dealt with here; the third one will be discussed later, because it is used for the analysis of our observations in Chorthippus and Leptysma.

As mentioned above, one first approach to detect interference was to compare the distribution of chiasma frequencies per chromosome with the Poisson expectations (Haldane, 1931). However, this approach is rather indirect. Even in the case of a good fit with a Poisson expectation (as found for a mutant genotype in rye by Jones, 1967) the operation of interference cannot be strictly ruled out because this method tells us what is going on between bivalents, whereas interference takes place within bivalents. The comparison of chiasma frequency in specific segments of a chromosome, be they adjacent or not, is a more direct approach to detecting interference. A negative correlation of chiasma numbers between segments is evidence of interference; a positive correlation indicates 'negative interference' (that the presence of one chiasma on one arm enhances the probability of chiasma formation in the other). Nil correlation means no interference, complete coincidence $(C=1)$ and hence complete independence between segments. And yet this method is not without shortcomings: it needs a marker between the chiasmata. In most bivalents there is a universal marker, the centromere. This restricts this analysis to meta- or submetacentric chromosomes. Harte (1956), in a detailed study of Paeonia tenuifolia, compared both arms of all five bivalents in this plant. Suggestively enough, in subtelocentric chromosomes the correlation of chiasma number on each arm was consistently negative, but only in the small metacentric was this negative correlation significant. No clear interference pattern emerged from the long metacentric bivalent. Using a similar approach, Pätau (1941) concluded that interference across the centromere existed in Culex pipiens and that in Dicranomya trinotata its presence was not so clear. It is true that chiasma frequency is much higher in the second species than in the first, so probably interference was much stronger in Culex than in Dicranomya and therefore easier to detect.

These studies highlight a major flaw in the method of correlating chiasma numbers in both arms: it does not take into account the positions of chiasmata (Jones, 1987). Sybenga \& de Vries (1972) examined two plants of rye bearing the 'same' metacentric B chromosome: one of them showed strong interference across the centromere, but the other did not. The authors concluded that this resulted from genetically conditioned differences in chiasma localization between plants: whereas one almost invariably carried one proximal chiasma in one of the arms of the metacentric, the other did not. Therefore, for this method (the correlation between chiasma numbers in both arms of a chromosome) to detect across-centromere interference, the occur- 
rence of a proximal chiasma is mandatory. Hence it seems that studies which take into account chiasma position in both arms should be more telling than those which only correlate chiasma number. In fact, Callan \& Montalenti (1947) had revisited C. pipiens, using Theobaldia longiareolata, another related species, as a control and demonstrated the existence of interference across the centromere in the first species but not in the second. The clearest evidence comes from their observations of chiasma position: they divided the arms into two regions, $\mathrm{P}$ and $\mathrm{D}$ (for proximal and distal). In bichiasmate bivalents the $\mathrm{P} / \mathrm{P}$ class was significantly deficient, whereas $\mathrm{D} / \mathrm{D}$ bivalents were in excess. No such pattern was visible for Theobaldia, where chiasma frequency was roughly twice as great. They concluded, as Pätau (1941) did, that in C. pipiens interference acts across the centromere, that this interference decreases as the distance between chiasmata increases, and that this was not detectable in Theobaldia because of weak interference. How weak is this interference? Sybenga (1975) re-examined Callan and Montalenti's published data and calculated an indirect measure of interference strength, given as a ratio between mean and variance of chiasma frequencies (in a Poissonian distribution, with no interference, the ratio should be 1). This ratio is 8.066 in Culex and 3.633 in Theobaldia; Sybenga (1975) concluded that interference was weaker in the latter, this being reflected in the higher chiasma frequency as well as in the failure to detect interference across the centromere in this species.

By far the most suitable organisms for assessing interference across the centromere are Truxaline grasshoppers with meta- or submetacentric chromosomes. Southern (1967) studied chiasma distribution in four Truxaline species, C. brunneus, Myrmeleotettix maculatus, $C$. parallelus and Omocestus viridulus, with the aim of testing Mather's sequential model, by the same approach as Henderson (1963) but in species with bi-armed chromosomes. He concurred with Henderson's (1963) interpretation of sequential chiasma formation towards the centromere on the grounds of the high incidence of distal chiasmata. As for interference across the centromere, unfortunately, he resorted to the method of correlating chiasma numbers in both arms of all three (sub)metacentrics of all four species. The species with the highest chiasma frequency, $O$. viridulus, also had the shortest interchiasma ('ix') distances (suggesting weak interference) and showed positive acrosscentromere correlation in the two longest metacentric bivalents (but suggestively not in the shortest). Instead correlation was always negative, if nonsigni- ficant, in the species with the lowest chiasma frequency and longest ' $i x$ ' distances, $C$. brunneus.

In this latter species a detailed and very accurate chiasma distribution study in a single bivalent was conducted by Laurie (1980; partially published in Laurie \& Jones, 1981). Chorthippus brunneus has three long submetacentric and five acro- or telocentric chromosome pairs, plus an $\mathrm{X} 0 / \mathrm{XX}$ sex determination mechanism. The third bivalent (L3) was chosen for this study because it is the only identifiable bivalent in which both within- and betweenarms chiasma distribution can be assessed (pairs one and two cannot be reliably distinguished from each other); the position of the centromere was unambiguous because of a C-positive pericentric heterochromatin knob. Chiasma position was carefully measured in both arms. For this study, the individuals came from a natural population and three experimentally bred families. Each family was the result of a single-pair mating between individuals from the natural population. In this case it was argued that, unlike previous studies (see previous section) across-centromere interference should be assessed not by correlating chiasma numbers in both arms, but rather by correlating the distance from the centromere to the 'first' (the most proximal) chiasma in each arm for every individual. In most cases correlation was not significant and sometimes positive, perhaps as a result of small sample sizes (the data from different individuals were not pooled) and other factors that will be discussed later. Nevertheless, we think the application of this method is one of the two most valid methods to detect chiasma interference cytologically (Jones, 1987). The other is the estimation of coincidence values by dividing the bivalents into $n$ units of arbitrary length, in the fashion of genetic studies, but using chiasma distribution analyses (Jones, 1984). We used the first method in $C$. brunneus and $L$. argentina and the second in $C$. brunneus alone. Both were consistent in detecting interference across the centromere. The calculation of coincidence values is sufficiently consecrated by tradition as a valid method to estimate interference, and so we will concentrate first on the correlation of distances approach as a valid method to assess interference.

\section{Correlation of centromere to chiasma distances as a valid measure of interference}

Interference, as a statistical concept, means a departure from orthogonality, from independence between two (or $n$ ) variables, and is usually assessed in terms of covariance and/or correlation coeffi- 
cients; its significance is measured by means of an analysis of variance (continuous approach). Instead, genetic interference, as first defined, is the reduced probability of a crossover taking place in the vicinity of another. This is not usually assessed in terms of correlation because genetic interference is assessed in discrete, rather than continuous, intervals, and hence its extent cannot be measured by means of covariance (the significance of which is evaluated with analysis of variance) but in terms of coincidence, which follows a chi-squared distribution.

What we see here is that statistical and genetic definitions of interference are not far apart, and that both of them point to the same phenomenon, a departure from independence. We will exploit this parallel in order to use correlation coefficients as a valid approach to interference. In so doing, we are not trying to put forward another definition of interference; as a departure of independence, it can be assessed by any suitable statistical device. In our case, we would like to stress that correlation may be a useful tool to detect interference by cytological means.

\section{Leptysma argentina}

In a study originally designed to assess the effect of a centric fusion on chiasma distribution, an evaluation of chiasma interference across the centromere was carried out in the South American grasshopper L. argentina (Colombo, 1993). This species has a basic karyotype consisting of one large submetacentric and nine telocentric pairs plus an $\mathrm{X} 0 / \mathrm{XX}$ sex determination system; this karyotype is modified by several polymorphisms, among them a centric fusion between pairs 3 and 6 that leads to the formation of three karyotypes: basic unfused homozygotes (BB); heterozygotes $(\mathrm{BF})$; and fusion homozygotes (FF) (Bidau \& Hasson, 1984). This centric fusion was shown to reduce chiasma frequency in carriers (BF and $\mathrm{FF}$ ), especially close to centromeres, across the entire genome (Colombo, 1990). Under the working hypothesis that the reduction of chiasma frequency was caused by an increase in interference strength, chiasma position was measured in 2805 arms from 255 cells from all three karyotypes (BB, BF and $\mathrm{FF}$ ). The linear sequential chiasma hypothesis, as modified by Fox (1973), was used as an interpretative tool (see above).

The working hypothesis was proven correct, but not as expected. It had been assumed that the CDM would trigger two chiasmata if the interference distance ' $i$ ' was short, but only one if ' $i$ ' was long enough to prevent the formation of another chiasma. This was supposed to be true for every arm, because both arms of submetacentrics 1 and $3 / 6$ were treated separately. Yet, despite marked and significant differences in mean and minimum interchiasma distances ( $\overline{i x}$ and $i x_{\min }$, respectively) between karyotypes, the chiasma frequency of telocentric bivalents was the same in all karyotypes. The only difference was that chiasmata tended to be further apart in BF individuals, and even more so in FF ones, as a signal of increasing interference. It was the submetacentrics 1 and $3 / 6$ which made all the difference (lower chiasma number and fewer proximal chiasmata). Hence the presence of interference across the centromere was assessed using the abovementioned (Laurie, 1980) approach, i.e. correlating the distance from the centromere to the nearest chiasma in each arm. However, correlations were nonsignificant (actually close to zero) in all three karyotypes when the entire data were included.

As previously mentioned, for two chiasmata to interfere they must be sufficiently close; if they are too far apart they will not interact at all (coincidence $=1$ ). In fact, chiasmata tend to occupy distal positions in $L$. argentina and consequently the majority of chiasmata are too distant from their nearest neighbours in the other arm for interference to operate. In order to avoid this effect an elementary computer program was devised so that all bivalents in which $i x$ distance across the centromere was greater than a fixed value were excluded from the calculations. If chiasma interference across the centromere was undetected because of the statistical 'noise' caused by the bivalents with long interchiasma distances, it was expected that the shorter the artificially imposed upper limit, the higher the negative correlation would be. In Fig. 2 the correlation coefficient is plotted against the fixed limit $i x$ values for bivalent 1 . We can see that when the limit $i x$ decreases (i.e. when the stringency of the test is stronger), the correlation value creeps up and becomes significant. We stated earlier that $i x_{\min }$ (a rough estimation of interference strength) is larger in $\mathrm{FF}$ and $\mathrm{BF}$ individuals than in $\mathrm{BB}$ ones. If this method to assess interference across the centromere is valid, then the curves for fusion carriers should be steeper. This is exactly what was observed (Fig. 2). The greater the stringency of the test (dealing only with bivalents with shorter $i x$ distances), the stronger the measure of interference.

\section{Chorthippus brunneus}

We also applied the same method to the impressive mass of data collected by Laurie (1980) on chiasma 


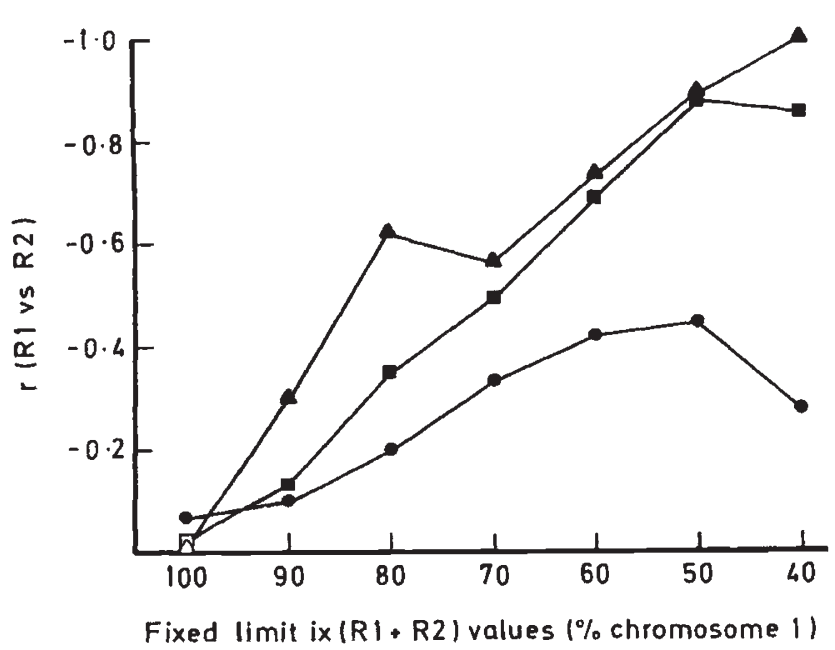

Fig. 2 Correlation coefficients $(r)$ between centromere to nearest chiasma in the long arm (R1) and in the short arm (R2) plotted against 'fixed limit' interchiasma distances (R1 + R2) for chromosome 1 of Leptysma argentina, in three different karyotypes BB (O), BF( $\square)$ and FF $(\triangle)$. See text for more detailed explanation. Solid (black) symbols indicate $r$-values which are significant at the 5 per cent probability point at least.

distribution in the submetacentric chromosome $\mathrm{L}_{3}$ of C. brunneus, by analysing 1467 bivalents (partially published in Laurie \& Jones, 1981). This sample of cells actually comes from 42 individual insects drawn from a natural population and three families reared in captivity. As mentioned above, the application of correlation analyses separately to every individual yielded inconclusive results (Laurie, 1980). In this present study, the data from 42 individuals were pooled. In this case the correlation between 'centromere to first chiasma' distances in both arms of all bivalents studied yielded a low negative correlation $(r=-0.201)$, which was, nevertheless, highly significant because of the large number of bivalents considered $(P<0.001)$.

Bivalent $\mathrm{L}_{3}$ of $C$. brunneus usually has, at most, three chiasmata (usually two in the long arm and one in the short one) or two at least (one in each arm in the vast majority of the cases): one-chiasmate bivalents are rare (1.34 per cent) and four-chiasmate even rarer $(0.47$ per cent). Given the large sample available here, we could afford in this case to divide it into two subsamples, one of trichiasmate and the other of bichiasmate bivalents. The trichiasmate subset of bivalents gave a significant negative correlation of distances from the centromere to the nearest chiasma in each arm $(r=-0.505$, $\left.t_{255}=9.388 ; P<0.001\right)$. This was expected, because, given the presence of three chiasmata, we were dealing with short interchiasma distances which did not vary very much (Fig. 3b). It comes as no surprise, then, that the narrowing down of the upper limit of $i x$ distance makes little difference when applied to this subset of bivalents (Fig. 4), because the $i x$ distance distribution is already rather narrow.

The case of bichiasmate bivalents is quite different, because here chiasma distribution is more relaxed (Fig. 3a). Hence there is plenty of room for $i x$ distance to vary from $i x_{\min }$ to almost the entire length of the bivalent. As expected, the correlation of centromere to chiasma distances was very low $(r=-0.135)$ when all bichiasmate (1:1) bivalents were included in the test, but even this was highly significant $(P<0.001)$ as a result of the large number
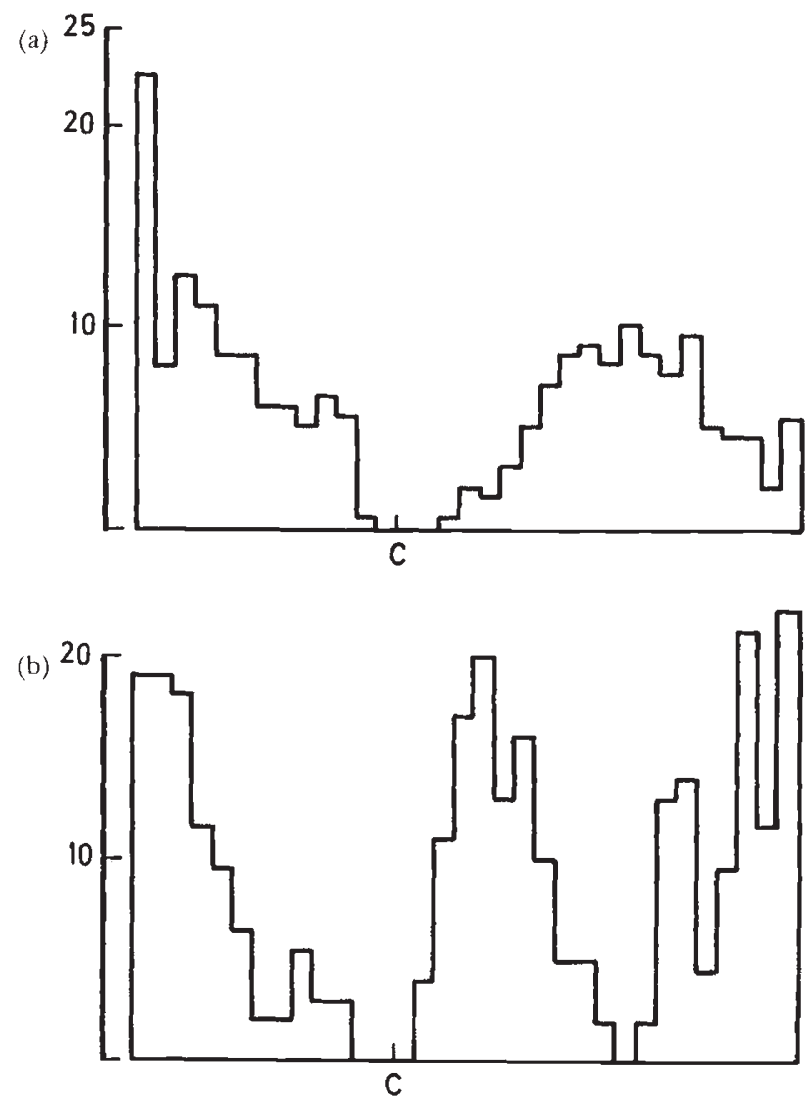

Fig. 3 Histograms showing the distributions of chiasmata in (a) 389 bichiasmate $(1: 1) \mathrm{L}_{3}$ bivalents of Chorthippus brunneus and (b) in 94 trichiasmate (1:2) bivalents. The data are normalized to chiasmata per 100 bivalents. These histograms are based on a sample of 511 bivalents from 13 full-sib individuals belonging to a single family; 18 bivalents having other combinations of chiasmata in the long and short arms are not included. For the purpose of this representation the bivalent is divided into regions corresponding to 5 per cent of the long arm; the position of the centromere (c) is indicated (from Jones, 1984). 


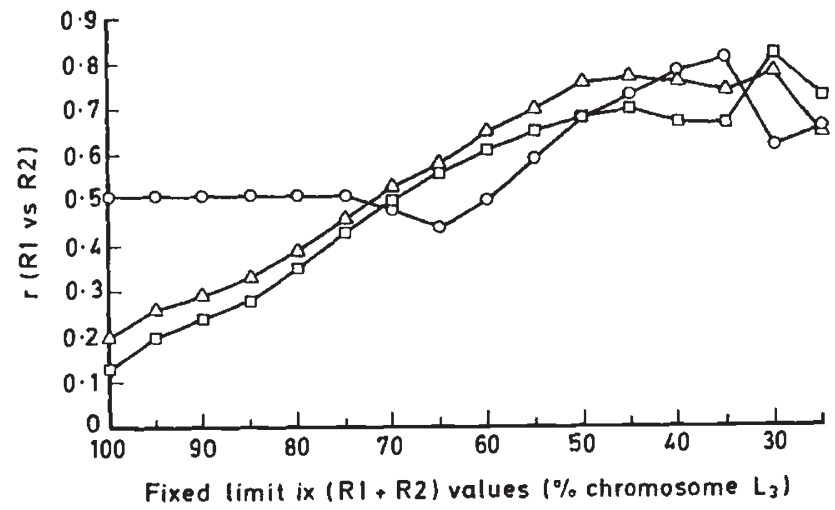

Fig. 4 Correlation coefficients $(r)$ between centromere to nearest chiasma in the long arm (R1) and in the short arm (R2) plotted against 'fixed limit' interchiasma distances ( $\mathrm{R} 1+\mathrm{R} 2$ ) for chromosome $\mathrm{L}_{3}$ of Chorthippus brunneus. $r$-values are plotted separately for trichiasmate bivalents $(\bigcirc)$, bichiasmate bivalents $(\square)$ and all bivalents $(\triangle)$. See text for more detailed information.

of bivalents involved. By narrowing down the upper limit of $i x$ distance the value of $r$ increases progressively until it reaches -0.8 for distances close to $i x_{\min }$ (Fig. 4). The joint analysis (all bivalents considered) is much closer to the result of bichiasmate bivalents, because this class is considerably more abundant.

These analyses leave little doubt that interference acts across the centromere in this species too. It is interesting to note, though, that in trichiasmate bivalents the other two factors which contribute to chiasma control, namely localization and number of chiasmata per bivalent, obscure the relative importance of interference. This is true even though correlations are significant and increasingly negative when the stringency increases.

\section{Interference is blind to centromeres}

These analyses demonstrate that interference acts across the centromere, and that the previous failure to detect it was caused, as is usually the case, by the presence of bivalents with chiasmata that were too far apart to interact at all or to insufficiently large samples of bivalents. The question is whether or not this interference across the centromere is different in any respect from within-arm interference.

In Fig. 5 we show a histogram of interchiasma distances across the centromere for all 1467 bivalents of $C$. brunneus. For the sake of comparison, interchiasma distances within the long arm are included: obviously only long arms with two chiasmata are considered here. In both cases $i x$ distances are expressed as percentages of chromosome 3 length. We see a relatively high frequency of bivalents with $i x$ distances across the centromere longer than 80 per cent of chromosome 3 length. As $i x$ distance across the centromere approaches $i x_{\min }$, the number of bivalents decreases steadily; the same is true for within-arm $i x$ distance. When finally they reach $i x_{\text {min }}$, between-arm and within-arm $i x$ values do so at the same point. There seems to be no quantitative difference between these two types of interference, because in both cases $i x_{\min }$ is 20 per cent of the chromosome length.

We repeated this comparison with $L$. argentina data for chromosome 1 (all three karyotypes were pooled here in order to obtain a respectable sample size). The pattern is strikingly similar (Fig. 6). We added to the comparison the $i x$ distances for the acrocentric chromosome 2, expressed as percentages of chromosome 1 length. We can see that there are many more bivalents with $i x$ distances across the centromere greater than 80 per cent of the chromosome length when compared to $C$. brunneus. All three variables peak at different lengths (we know that mean ix distance depends not only on inter-

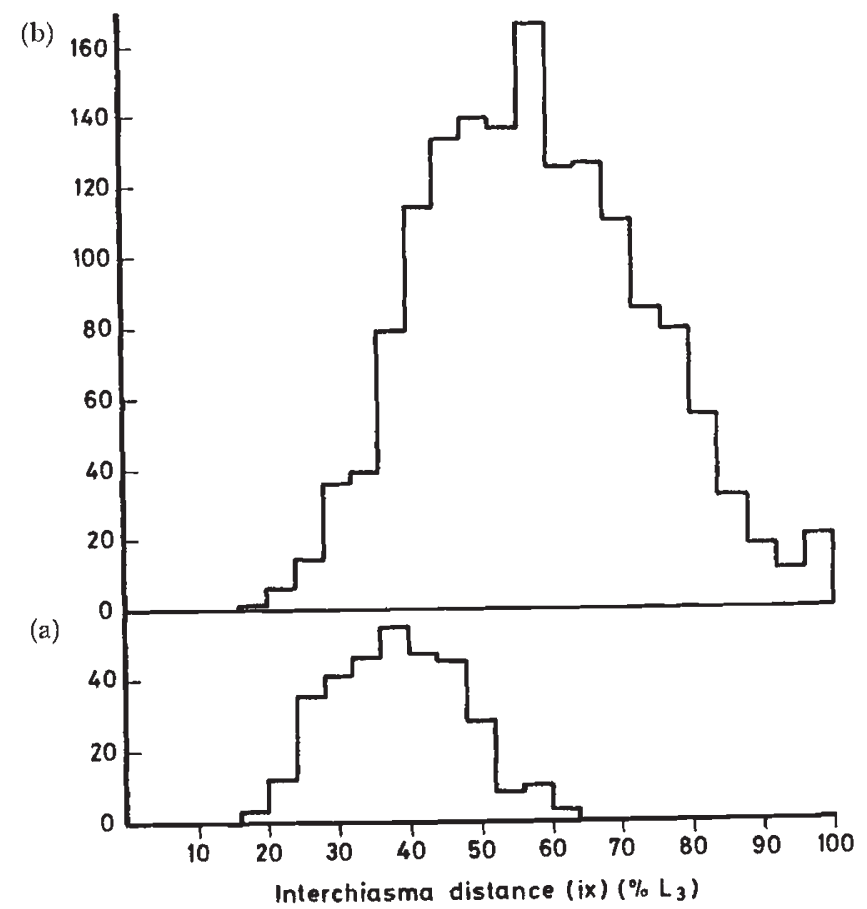

Fig. 5 Frequency histograms to show the distributions of interchiasma distances $(i x)$ in the $\mathrm{L}_{3}$ chromosome of Chorthippus brunneus, expressed as percentages of total chromosome length. Histogram (a) shows the distribution of $i x$ within the long arm, and histogram (b) shows the distribution of $i x$ values across the centromere. 


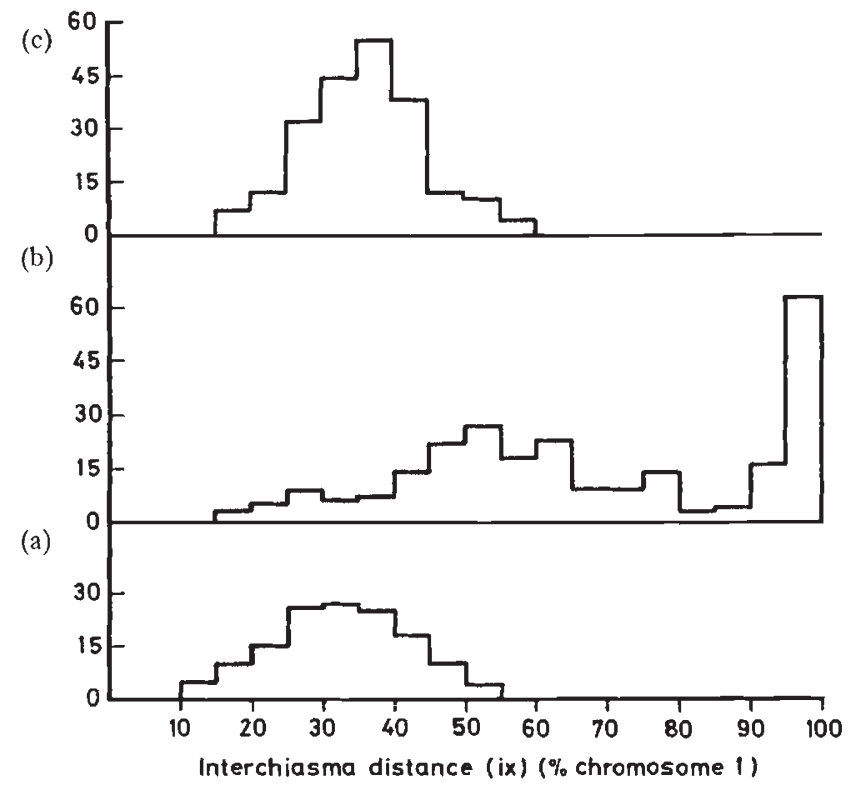

Fig. 6 Frequency histograms to show the distributions of interchiasma distances in Leptysma argentina, (a) within the long arm of chromosome 1, (b) across the centromere of chromosome 1 and (c) in the acrocentric chromosome 2. All $i x$ distances are expressed as percentages of the total length of chromosome 1.

ference but also on the remaining length of the bivalent) but all end at the same point ( 15 per cent of chromosome length) with convincing unanimity, suggesting again that interference across the centromere and interference within arms are the same thing.

\section{Detecting interference across the centromere by calculation of coincidence}

So far we have used a continuous approach to assess interference. We can turn back to the timehonoured expedient of calculating coincidence values, in this case using chiasma distribution, rather than genetic, data. This method was used by Newcombe (1941) in metaphase I chromosomes of Trillium erectum, but given the highly condensed nature of the chromosomes in this stage the conclusions are far from reliable. Much more reliable are the data from C. brunneus taken at diplotene.

The procedure is as follows. In genetic studies we have markers, and measure the frequency of recombination between markers. In the long diplotene chromosomes of Orthoptera we can divide the bivalents arbitrarily into as many equal segments as may be conveniently handled; so, we have as many 'markers' as we wish, only that these markers are given by units of length or, to accommodate variations of chromosome contraction, units of relative length. For this analysis the long arm was subdivided into 10 equal intervals, and taking one long arm interval as a standard unit of relative length, the short arm was subdivided into seven intervals. Each interval was recorded as containing, or not containing, a chiasma in each of $1467 \mathrm{~L}_{3}$ bivalents (a single interval never contained more than one chiasma), and these observations were used to estimate the overall mean chiasma frequency of each interval. These interval mean chiasma frequencies were then used to calculate the expected frequencies of double chiasmata for each pair of intervals. Because the entire chromosome is divided into 17 intervals, the number of different pairwise combinations of intervals is $\left(17^{2}-17\right) / 2=136$. Coincidence, then is calculated in the usual way as the ratio of actual (observed) double chiasmata to the expected numbers, for each pair of intervals (Table 1). This approach was carried out in $C$. brunneus for withinarm interference (Jones, 1984) and in the present study this approach was extended to interference across the centromere using the data of Laurie (1980). This study leads, from another perspective, broadly to the same conclusions as the correlation approach.

Confining our attention initially to the long arm only, it is evident that interference is complete over distances equalling 25-30 per cent of the arm, because pairs of intervals separated by only one or two other intervals show coincidence values of zero (Table 1, Fig. 7a). Over longer distances, interference continues to be expressed for a further 30 per cent of the arm, but decreases gradually over this distance until intervals which are separated by five other intervals show coincidence values of about 1 . An interesting phenomenon, already noticed by Jones (1984), is that sometimes coincidence rises to values higher than one in regions six to eight intervals apart, yielding apparent negative interference. This means that the formation of a chiasma in one region, increases the probability of chiasma formation in another. This is as might be expected when chiasma distribution is crammed (see Fig. 3) and there is: (i) a high degree of localization (most chiasmata are distal and proximal); and (ii) a tight control of chiasma frequency (most chromosomes have two or three chiasmata). The occurrence of one chiasma in a distal region then enhances the probability of chiasma formation in a proximal region of the same arm not despite interference but rather because of it, coupled with the other two factors mentioned earlier. 
To investigate interference across the centromere, we repeated the above procedure, but confined the analysis to pairs of intervals from different arms, that is, one short arm interval and one from the long arm (Fig. 7b). Once again we find zero or low coincidence values for adjacent or closely spaced intervals, demonstrating unequivocally the operation of interference across the centromere. In agreement with within-arm comparisons, coincidence increases to 1 for regions five or more intervals apart. It may also increase to values higher than 1 for regions six to nine intervals apart, which we interpret as yet another indication of localization and chiasma number control. We want to stress that both the number of intervening regions required to reach coinci- dence values of 1 , and the unexpected rise to values higher than 1, are the same for the between-arm as for the within-arm comparisons. Again, we cannot see any difference in the operation of interference between or within arms, reinforcing the idea that the centromere is ignored by interference.

Another interesting feature is that there seems to be 'interference' between chiasmata at both extremes of the chromosome, sometimes 13-14 regions apart. Obviously this cannot be caused by direct interaction, for these chiasmata are too far apart to interact. What happens is that, because of the crowding of chiasmata, distals in the long arm interfere with proximals in the long arm, and these in turn interfere with the chiasmata in the short arm.

(a)

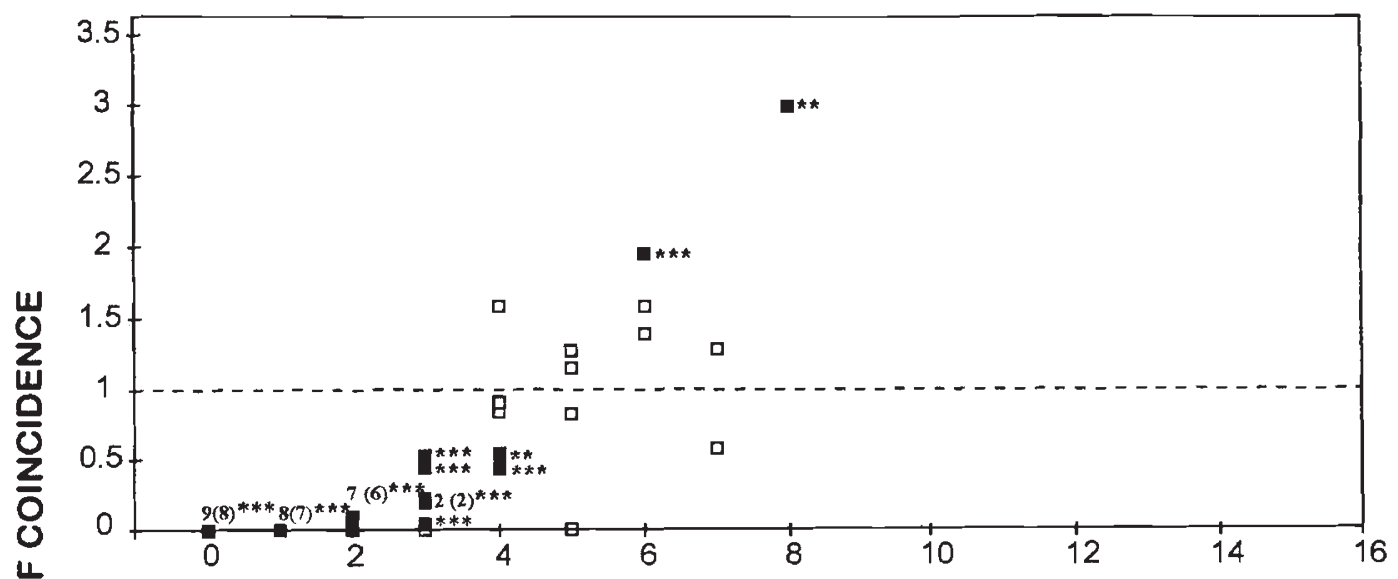

(b)

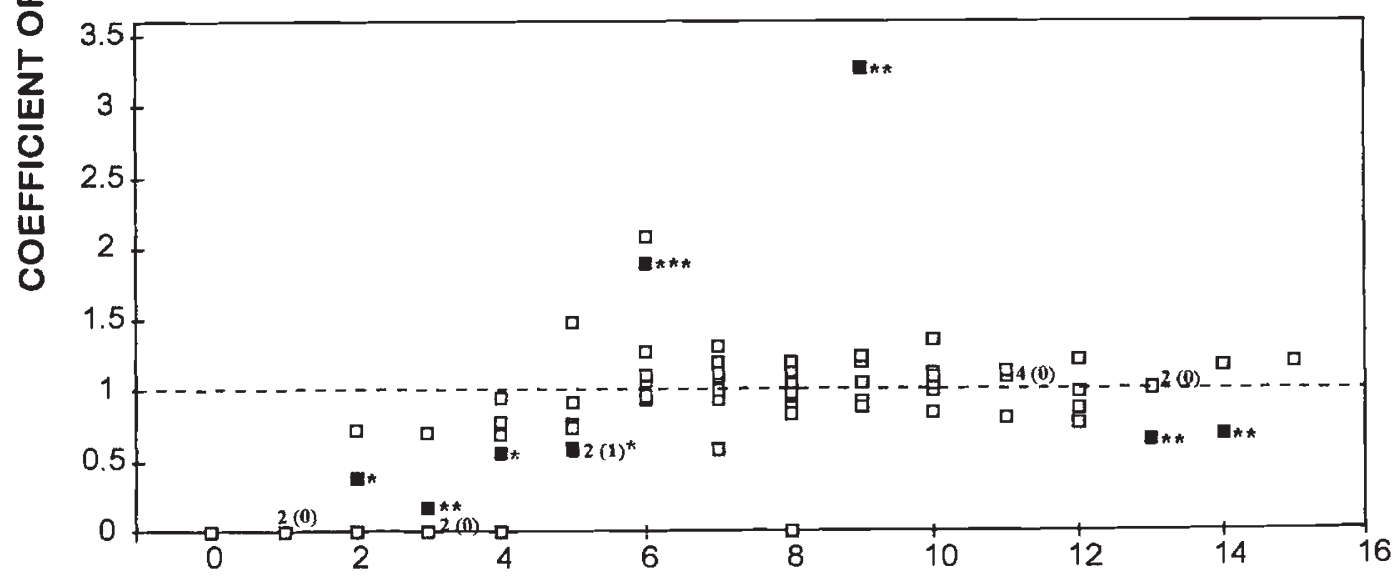

NUMBER OF INTERVALS

Fig. 7 Coefficients of coincidence for pairwise combinations of chromosome intervals in the $\mathrm{L}_{3}$ bivalent of Chorthippus brunneus, plotted against the numbers of intervals separating each pair of intervals being considered. (a) The plot for pairs of intervals within the long arm.(b) The plot for pairs of intervals in the short and long arm, that is across the centromere. Data for the short arm only are not shown because double chiasmata in the short arm are very infrequent. Values of the coefficient of coincidence which are significantly less, or greater, than unity are shown ( $\square$ ) and the significance level is indicated by asterisks $\left({ }^{*} 0.05 ;{ }^{* *} 0.01 ;{ }^{* * *} 0.001\right)$. When two or more values coincide, the numbers of overlapping values are shown numerically, and the numbers of significant values are given in parentheses. 


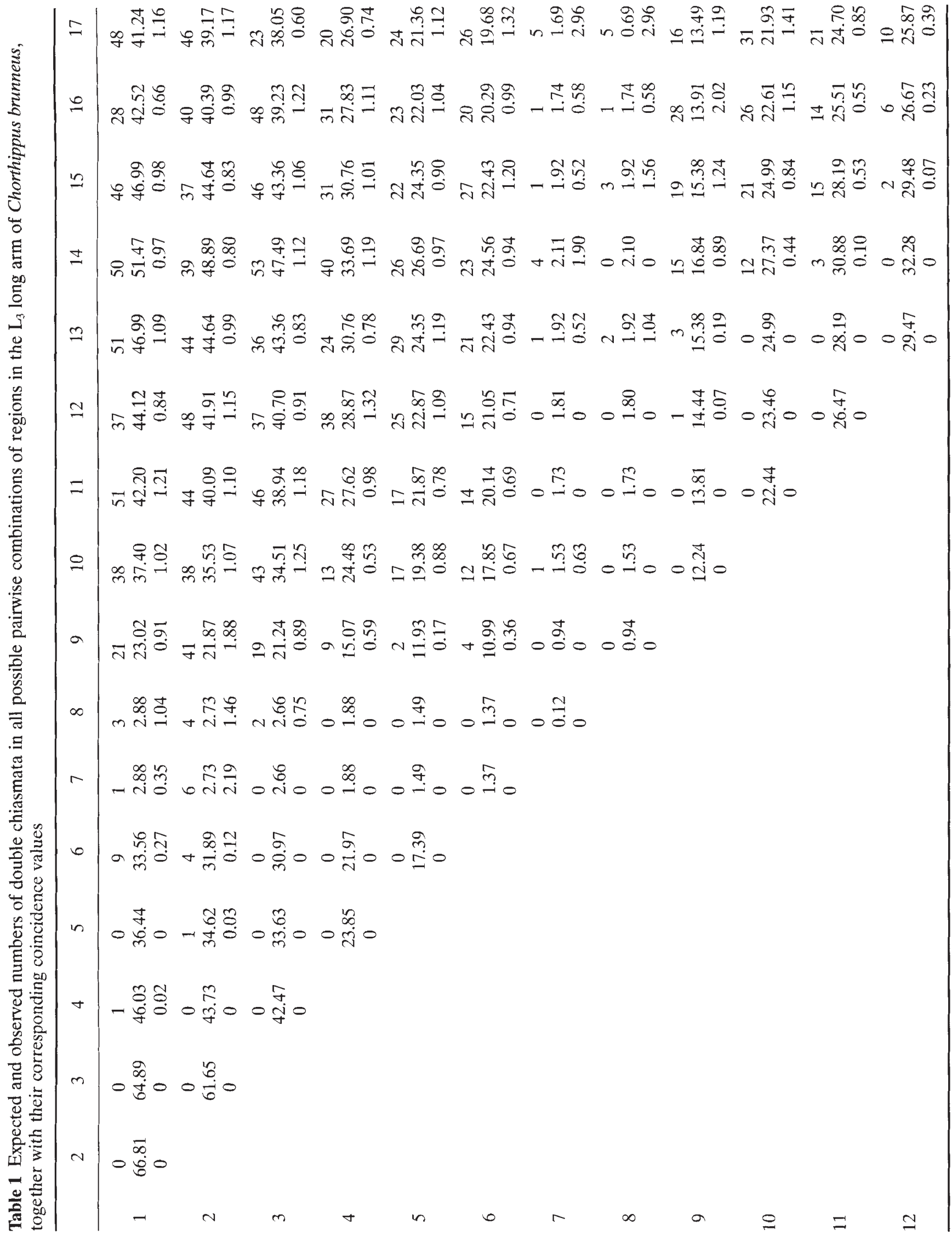




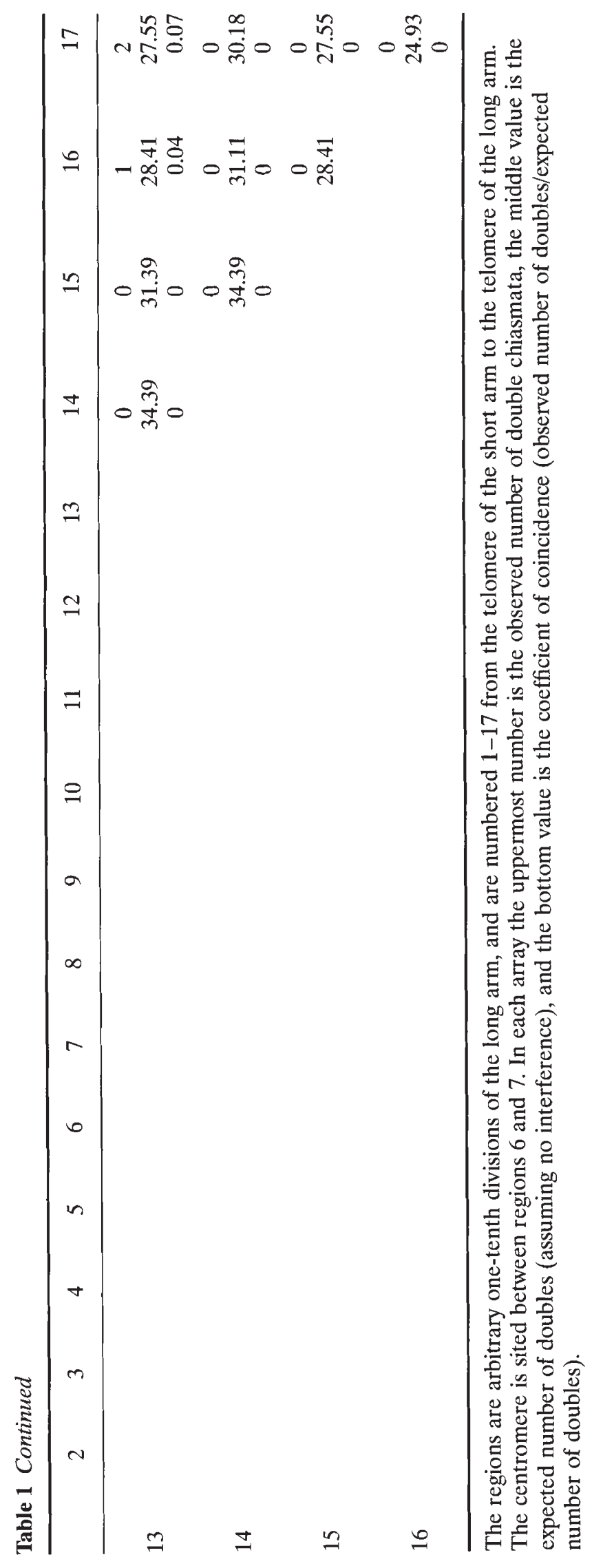

(c) The Genetical Society of Great Britain, Heredity, 79, 214-227. 
This is another dramatic example of interference across the centromere.

\section{Conclusions}

Mather (1936a), on the basis of evidence accumulating rapidly at that time, was convinced that the centromere had an important role in the determination of chiasma position; it has. Chiasma formation is mindful and respectful of the centromere, for the configuration of the bivalent in metaphase and, consequently, the fate of meiosis as a whole depends on it. Among cases of absolute localization there are more cases of distal, rather than proximal localization (John, 1990), perhaps because this ensures a maximum of elasticity of the bivalent in metaphase I. This is no obstacle to complete proximal localization in other cases, however. In many other organisms we notice a tendency of chiasmata to steer clear of the centromere and to occur more frequently in distal locations. From this clear influence of the centromere on chiasma position, Mather (1938) concluded that it had a causal influence in their formation and that, starting from the centromere, a wave of chiasma formation proceeds towards the telomeres. This logical leap, in our opinion, seems unjustified (Colombo, unpublished observations). Nonetheless, it led to the development of a sequential model of chiasma formation starting at the centromeres, and this would necessarily mean that both arms were independent of each other. Mather quoted all the evidence he could find in favour of his hypothesis, which had the appeal of simplicity and linearity. Suggestions of nonindependence between arms (Graubard, 1932; Schweitzer, 1935) were dismissed (Mather, 1936a) based on studies performed by Stevens (1936). This controversy belongs entirely to the history of science now; what is really important is the fact that both arms were perceived to be independent, because this hypothesis was central to Mather's model. Now we have tried to gather as much information as we can to prove that the centromere, however important in the determination of chiasma position, is no barrier to interference. All it can do is to prevent chiasmata forming in its vicinity (although in some species it encourages them); what it cannot prevent is the 'transmission' of interference.

The purpose of this review is to demonstrate, first, that the centromere is no barrier to interference; and, secondly, that interference does not see it. Some consequences arise from this disregard of interference to centromeres. The most important of them all, however, seems to be the fact that it renders the linear sequential model of chiasma formation difficult to support. It requires independence between arms, even if, as argued by Henderson (1963), Southern (1967), Fox (1973), Hultén (1974) and Colombo (1993), the sequence was deemed to proceed towards the centromere. Now the model has become too heavy and unparsimonious to maintain. If chiasma formation does not start from the centromere, or end against the centromere, where does it start from or end? Or, to put it bluntly, does it start from anywhere?

\section{Acknowledgements}

This study was conducted while P.C.C. was in receipt of a Fellowship granted by the Consejo Nacional Científicas y Técnicas (CONICET). We are grateful to David Laurie for his consent to plunder his unpublished Chorthippus brunneus chiasma data in his $\mathrm{PhD}$ thesis. We also thank our colleague Paul Fransz for wrestling with the computer to produce Fig. 7.

\section{References}

BEADle, G. W. 1932. A possible influence of the spindle fibre on crossing-over in Drosophila. Proc. Natl. Acad. Sci. U.S.A., 18, 160-165.

BIDAU, C. J. AND HASSON, E. R. 1984. Population cytology of Leptysma argentina Bruner (Leptysminae: Acrididae). Genetica, 62, 161-175.

CAllan, H. G. 1949. Chiasma interference in diploid, tetraploid and interchange spermatocytes of the earwig Forficula auricularia. J. Genet., 49, 209-213.

CALlan, H. G. AND MONTALENTI, G. 1947. Chiasma interference in mosquitoes. J. Genet., 48, 119-134.

CHARLES, D. R. 1938. The spatial distribution of crossovers in X-chromosome tetrads of Drosophila melanogaster. J. Genet., 36, 103-126.

COLOMBO, P. C. 1990. Effects of centric fusions on chiasma frequency and position in Leptysma argentina (Acrididae: Orthoptera). II. Intra- and interchromosomal effects. Caryologia, 43, 131-147.

соцомво, Р. C. 1993. A polymorphic centric fusion enhances chiasma interference in Leptysma argentina (Orthoptera): a chiasma distribution study. Heredity, 70, 254-265.

FOSS, E., LANDE, R., STAHL, F. W. AND STEINBERG, C. M. 1993. Chiasma interference as a function of genetic distance. Genetics, 133, 681-691.

FOX, D. P. 1973. The control of chiasma distribution in the locust, Schistocerca gregaria (Forskal). Chromosoma, $\mathbf{4 3}$, 289-328.

GOWEN, J. W. 1919. A biometrical study of crossing-over. Genetics, 4, 205-250.

graubard, M. 1932. Inversion in Drosophila. Genetics, 17, 81-106.

(c) The Genetical Society of Great Britain, Heredity, 79, 214-227. 
HALDANE,J. B. S. 1931. The cytological basis of genetic interference. Cytologia, 3, 54-65.

HARTE, C. 1956. Die variabilität der Chiasmenbildung bei Paeonia tenuifolia. Chromosoma, 8, 152-182.

HENDERSON, S. A. 1963. Chiasma distribution at diplotene in a locust. Heredity, 18, 173-190.

HUltén, M. 1974. Chiasma distribution at diakinesis in the normal human male. Hereditas, 76, 55-78.

JoHn, B. 1990. Meiosis. Cambridge University Press, Cambridge.

JONES, G. H. 1967. The control of chiasma distribution in rye. Chromosoma, 22, 69-90.

JONES, G. H. 1984. The control of chiasma distribution. Symp. Soc. Exp. Biol., 38, 291-320.

JONES, G. H. 1987. Chiasmata. In: Moens, P. B. (ed.) Meiosis, pp. 213-244. Academic Press, Orlando, FL.

KING, J. S. AND MORTIMER, R. K. 1990. A polymerization model of chiasma interference and corresponding computer simulation. Genetics, 126, 1127-1138.

LANDE, R. AND STAHL, F. W. 1993. Chiasma interference and the distribution of exchanges in Drosophila melanogaster. Cold Spring Harbor Symp. Quant. Biol., 58, $543-552$.

LAURIE, D. A. 1980. Inter-individual Variation in Chiasma Frequency and Chiasma Distribution. A Study of Chorthippus brunneus and Man. Ph.D. Thesis, University of Birmingham.

LAURIE, D. A. AND JONES, G. H. 1981. Inter-individual variation in chiasma distribution in Chorthippus brunneus (Orthoptera: Acrididae). Heredity, 47, 409-416.

MAEDA, T. 1930. On the configurations of gemini in the pollen mother cells of Vicia faba L. Mem. Coll. Sci. Kyoto Univ. B., 5, 127-137.

MATHER, K. 1933. The relations between chiasmata and crossing-over in diploid and triploid Drosophila melanogaster. J. Genet., 33, 243-259.

MATHER, K. 1936a. The determination of position in crossing-over. I. Drosophila melanogaster. J. Genet., 33, $207-235$.

MATHER, K. 1936b. Competition between bivalents during chiasma formation. Proc. R. Soc. B, 120, 208-227.

MATHER, K. 1937. The determination of position in crossing-over. II. The chromosome length-chiasma frequency relation. Cytologia Fujii Jubilee Vol., 514-526.
MATHer, K. 1938. Crossing-over. Biol. Rev., 13, 252-292.

MATHER, K. 1939. Crossing-over and heterochromatin in the X-chromosome of Drosophila melanogaster. Genetics, 24, 413-435.

MATHER, K. 1940. The determination of position in crossing-over. III. The evidence of metaphase chiasmata. $J$. Genet., 39, 205-223.

MORgan, T. H. 1919. The Physical Basis of Heredity. J.B. Lippincott, Philadelphia, PA.

MUller, H. G. 1916. The mechanism of crossing-over. Am. Nat., 50, 193-221.

MUNZ, P. 1994. An analysis of interference in the fission yeast Schizosaccharomyces pombe. Genetics, 137, 701-707.

NEwCOMBE, H. B. 1941. Chiasma interference in Trillium erectum. Genetics, 26, 128-136.

PÄTAU, K. 1941. Cytologischer Nachweis einer positiven Interferenz über das Centromer (Der Paarungskoeffizient. I). Chromosoma, 2, 36-63.

sAX, K. 1930. Chromosome structure and the mechanism of crossing-over. J. Arnold Arboretum, Harvard, 11, 193-220.

SAX, K. 1932. The cytological mechanism of crossing-over. J. Arnold Arboretum, Harvard, 13, 180-212.

SCHWEITZER, M. D. 1935. Analytical study of crossing-over in Drosophila melanogaster. Genetics, 20, 497-528.

SOUTHERN, D. I. 1967. Chiasma distribution in Truxaline grasshoppers. Chromosoma, 22, 164-191.

STEVENS, N. M. 1936. The analysis of interference. J. Genet., 32, 51-64.

STURTEVANT, A. H. 1915. The behaviour of the chromosomes as studied through linkage. Z. für induk. Abst. Vererb., 13, 234-297.

SyBenga, J. 1975. Meiotic Configurations. Springer Verlag, Berlin.

SYBENGA, J. AND DE VRIES, J. M. 1972. Chromosome pairing and chiasma formation in polysomic B-chromosomes in rye, Secale cereale. Biol. Zentralbl., 91, $181-192$.

WEInstein, A. 1918. Coincidence of crossing over in Drosophila melanogaster (ampelophila). Genetics, 3, 135-172.

WHITEHOUSE,H. L. K. 1965. Towards an Understanding of the Mechanism of Heredity. Edward Arnold, London. 